\title{
Hardware Software Codesign of a Safety-Critical Embedded Computer System for an Automatic Endoscope
}

\author{
Gul N. Khan and Matthew Jin \\ Electrical and Computer Engineering, Ryerson University, \\ 350 Victoria Street, Toronto, Ontario, Canada M5B $2 \mathrm{~K} 3$ \\ gnkhan@,ee.ryerson.ca
}

\begin{abstract}
Hardware-software codesign is presented for a safety-critical embedded computer system. The system is applied for endoscope control and navigation. The embedded system architecture provides high performance computing for real-time implementation of machine vision algorithms and fault-tolerance for patient safety. It consists of five processor cores, local memory, I/O interface and multi-port shared memory. The hardware and software system architectures are codesigned. A virtual hardware is developed to execute the application and system software tasks. The system is designed and modeled sing VHDL and Eaglei toolset. We have limited system verification to co-verification of system hardware architecture and fault-tolerance strategies. Co-verification results indicate that the system performance degrades gracefully under various fault scenarios.
\end{abstract}

Keywords: Hardware-software codesign, High performance embedded systems; Automatic endoscope.

\section{INTRODUCTION}

The applications of embedded computer systems range from sophisticated aircraft flight control to consumer electronics. The high volume embedded applications include home appliances, automobiles, parking systems, cellular phones, toys, and many other devices that contain some sort of $\mu$-controller. On the other extreme are safety-critical applications like fly-bywire aircrafts, high-rise building elevators and medical instruments. The essential features of safety-critical embedded systems like endoscope are high performance, fault-tolerance and adaptability of the system. The architects of such system are facing high throughput and reliability demands that have never before been required of these systems. The system architecture has to provide fault-tolerant hardware support that can be programmed to implement software fault-tolerant strategies $[1,2]$.

The design of embedded system has adapted the brute force approach in the past. Hardware and software were designed separately while correctness and compatibility of two domains are sorted at the integration stage. Hardware-software integration problems lead to either large number of design iterations or non-optimal system architecture. Hardware-software codesign approach speeds up an intuitively serial design process by developing hardware and software concurrently. It is best suited to embedded system design and helps system designers to meet the design and development deadlines $[3,4]$. Hardware-software codesign shortens the development cycle, minimize bugs, manage cost, and produce competitive embedded systems.

The high performance embedded system presented in this paper is employed for endoscope control. Endoscope is a medical instrument used for diagnosing UGI, colon and bronchus diseases as shown in Figure 1. We have been concentrating on the automation of colonoscopy. It requires high performance computation for real-time implementation of machine vision algorithms and faulttolerance support for patient safety. High performance computing and fault-tolerance can be accomplished by a hardware engine of heterogeneous multiple processor. During conventional colonoscopy, the consultant advances the endoscope progressively while controlling its tip by judging the direction from a stream of colon images. The deepest area in colon (lumen) corresponds to the dark region near the center of colon images as shown in Figure 2. Lumen is an important navigational landmark for colonoscope control and navigation. We have investigated a number of methods to identify the lumen in the past [5]. Magnetic 3D imaging is also being introduced to support doctors during colonoscopy procedures [6]. Attempts are also made to navigate the endoscope using collision avoidance technique [7]. 


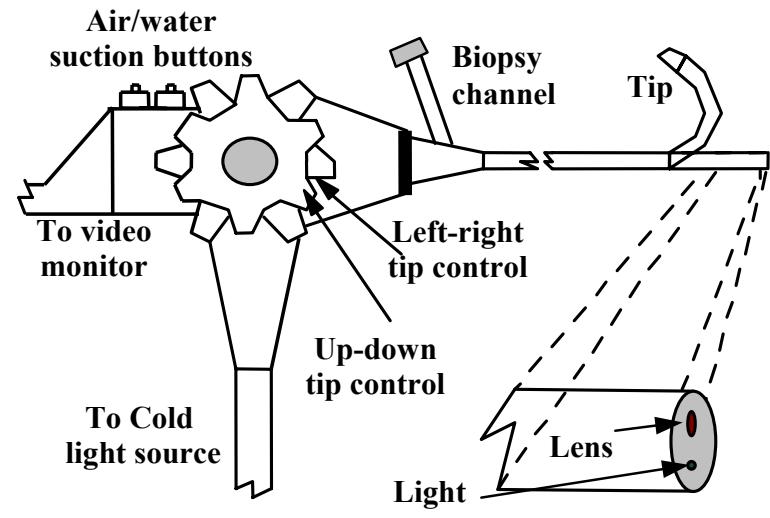

Fig. 1. Conventional endoscope

We have employed standard industrial and parallel computer systems for endoscope navigation in the past

[5]. However, it is observed that a dedicated embedded system is needed for such safety-critical applications. The main objectives of our research are to develop a fault-tolerant and high performance embedded system architecture. The software architecture to be realized by the embedded system includes task scheduling, interprocessor communication, image feature extraction and building a search space representation of colon.

\section{THE EMBEDDED SYSTEM}

A block diagram of the endoscope embedded computer system is illustrated in Figure 3. The system consists of an embedded computer and I/O modules. Digital outputs control the endoscope tip while highspeed analog input channels acquire video/ultrasound images of colon.

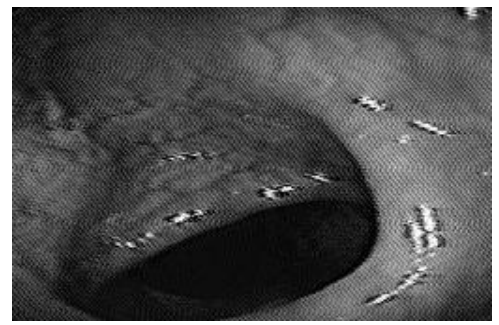

Fig. 2. Typical colon images

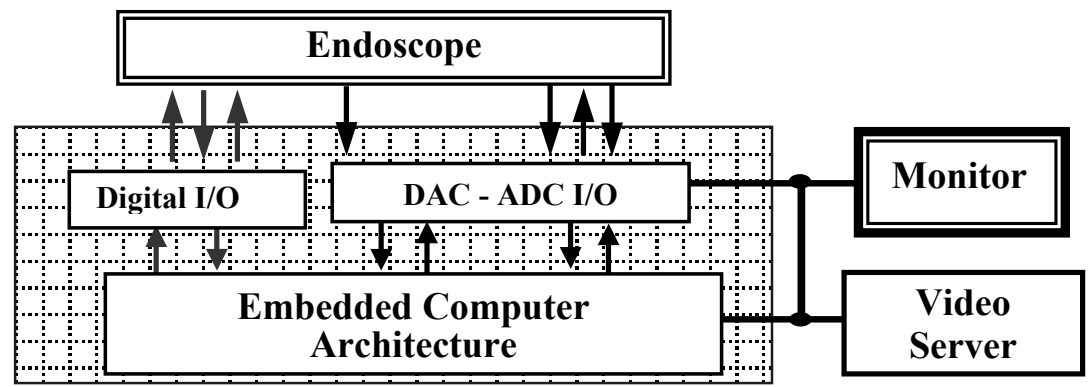

Fig. 3. Endoscope control embedded system

\subsection{Embedded Computer Architecture}

The embedded computer system consists of five nodes, which are fully connected by ten dual port memories $\mathbf{D P} \mathbf{P}_{\mathbf{i j}}$ as shown in Figure 4. High performance interconnection network provides efficient interprocessor communication. There are three compute processor cores $\left(\mathbf{W P}_{\mathbf{i}}\right)$ for computational intensive tasks and two IO processor cores $\left(\mathbf{I P}_{\mathbf{X}}\right)$ for real-time control.
The IP processors support fault tolerance and recovery from failures in addition to task scheduling and load balancing. Both IP processors are capable of serving as system controller, however at a given time, one of them is designated as controller for system monitoring. The other IP monitors the designated controller and take over the role of controller when the designated controller fails.

The system nodes are self-checking and in the case of a failure they isolate themselves. A watchdog timer 
detects a node failure while failure of DP memories and their interface is detected during message transfer. The processor interconnection network provides alternate routes for inter-processor communication. A number of fault-tolerant strategies are used for fault detection, containment and system recovery. The failure of node components is handled as a single fault. The interrupting capabilities of a faulty processor is disabled and its access to DP memories is also inhibited. The system controller broadcasts the failure to healthy nodes and invokes a diagnostic process. The failed node is put into service for a transient fault. Otherwise, it is kept out of the system and its tasks are re-scheduled. A node with a faulty program memory is utilized in a degraded mode by executing its critical tasks from DP memory blocks.

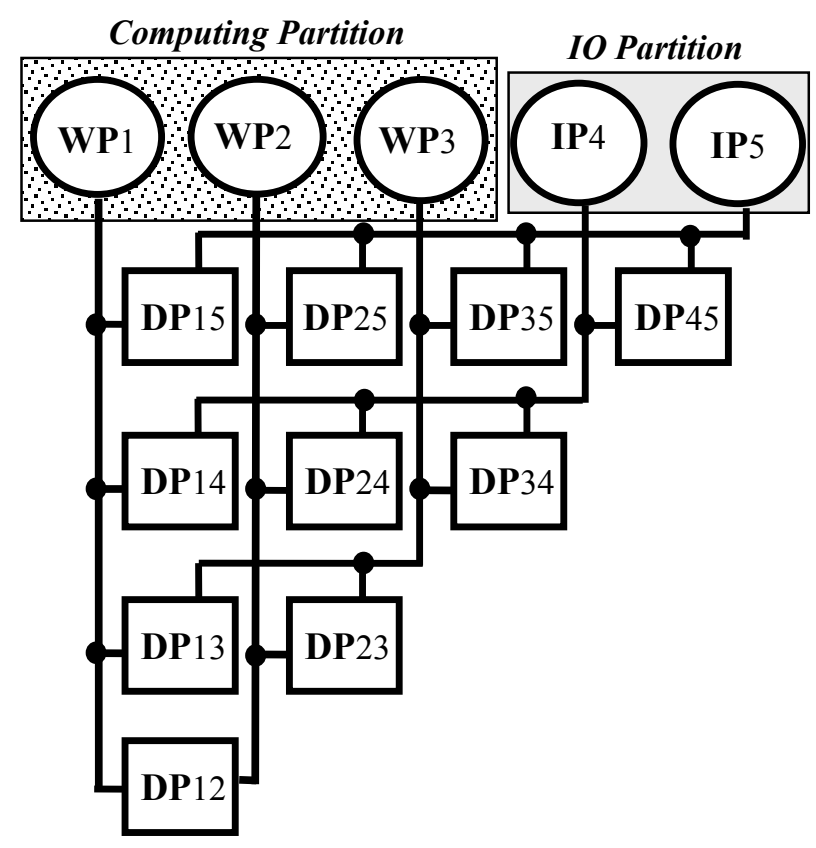

Fig. 4. Embedded processor architecture

The processor architecture supports various software and hardware fault-tolerance configurations. Three compute processors can be connected in TMR (triple modular redundant) mode while one of the IP processor serves as a voter. The other IP processor provides the input data to the TMR module as shown in Figure 5a. The architecture is flexible and one can configure both IP processors as a duplex voter for the TMR configuration as shown in Figure 5b.

\subsection{Endoscope Control}

The embedded system processor architecture can be envisaged having two partitions: computing and IO as illustrated in Figure 4. IO partition interfaces with endoscope sensors and issues tip movement commands. IO partition digitizes colon images and passed on to computing partition. Computing partition executes machine vision algorithms, detects the lumen and builds a search space representation. IO partition generates digital pulses for two motors for controlling endoscope tip in two concentric perpendicular directions. The tip direction movements are fed back to IO partition using analog input channels. Left-right and up-down tip movements are first translated into voltages that are finally converted by analog to digital conversion module. The feedback of tip movements forms a servo control mechanism where the servo loop is completed by a software process being executed by IO partition.

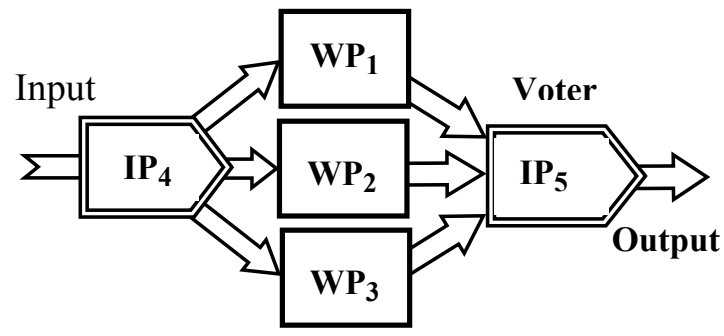

(a) Triple Modular redundancy

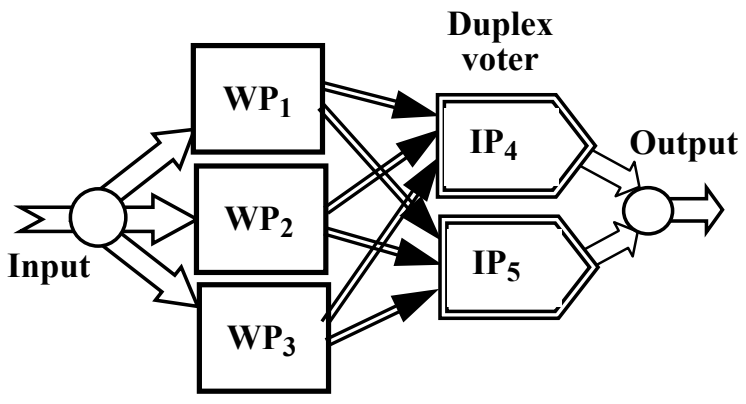

(b) Duplex voter

Fig. 5. TMR configurations

\subsection{Hardware Software Codesign}

Hardware-software co-design problem can be broken up into design specification, hardware/software partitioning and estimation of system performance. The design specification for describing system level behavior is a challenging problem. It needs high abstraction yet requires fine details to reduce ambiguities during synthesis. Embedded system is partitioned by using design constrains in terms of cost, power consumption, silicon size, speed, etc. After partitioning, the design comes down to hardware-software co-simulation. A cosimulation tool, Seamless uses instruction set simulator 
and a target processor model to create a virtual hardware environment. A more abstract approach is used by Eaglei to simulate the functional behavior of the system.

We have employed Eaglei for hardware-software coverification of the embedded system. The software execution environment interacts with a VHDL based hardware model having Virtual Software Processor (VSP). VSPs are functional description of $\mu$-processor cores divided into two halves that communicate with the hardware and software design entities. It allows the fetch/execute cycles to be handled by the software application running at the workstation speed, thereby freeing the HDL simulator to handle rest of the hardware on demand from the software application. Two types of processor cores: Oak DSP for computing and ARM7TDMi for IO partition. Other hardware modules are implemented in VHDL. System and application software tasks are coded in $\mathrm{C}$ language.
The main components of the IO and compute nodes include a virtual software processor, watchdog timer, private and dual-port memory interfaces. IP processors also contain processor-timer one each for the rest of system nodes that helps IP nodes to monitor other system nodes. A system status is kept at each node in a $4 \times 52-D$ register. The contents of status register, kept at IP5 are shown in Table 1. The 'Available' column field when set to ' 1 ' represents the node availability. The "Busy" field when set indicates a busy node that is unable to accept new tasks. The 'Reset' field when set implies that the node is currently being diagnosed. The 'TaskID' field provides the tasks details a node is executing. The node status register given in table indicates that IP4, WP1 and WP3 are available, DP15 is faulty; WP1 and WP3 are processing tasks 3 and 2 respectively.

Table 1. Status Register of IP5

\begin{tabular}{|c|c|c|c|c|c|}
\hline $\begin{array}{c}\text { System } \\
\text { Node }\end{array}$ & $\begin{array}{c}\text { Available } \\
(\mathbf{1} / \mathbf{0})\end{array}$ & $\begin{array}{c}\text { Busy } \\
\mathbf{( 1 / 0 )}\end{array}$ & $\begin{array}{c}\text { Reset } \\
\mathbf{( 1 / 0 )}\end{array}$ & $\begin{array}{c}\text { Availability } \\
\text { Com-module (1/0) }\end{array}$ & $\begin{array}{c}\text { Task } \\
\text { ID }\end{array}$ \\
\hline IP4 & 1 & 1 & 0 & 0 & - \\
\hline WP1 & 1 & 1 & 0 & 1 & 3 \\
\hline WP2 & 1 & 0 & 1 & 0 & - \\
\hline WP3 & 1 & 1 & 0 & 0 & 2 \\
\hline
\end{tabular}

\section{TESTING AND VERIFICATION}

A subset of endoscope control application is selected to verify fault-tolerance features of system architecture. It consists of a suit of matrix manipulation algorithms that are the basic building block of image analysis. The application is partitioned into tasks to be executed by compute processors. IP node provides support for faulttolerance, task scheduling and endoscope control. A dedicated process that executes concurrently along with application tasks facilitates fault-injection. We present the verification results of a few representative failure scenarios. One of the healthy IP nodes (IP4) becomes the system controller while the other takes the role of backup controller (IP5) at startup. Figure 6 shows the verification of system startup where each node checks itself and test the integrity of its DP memories.

A permanent fault is simulated by injecting a fault in WP1 node, in the next fault-scenario being presented in Figure 7. The controller (IP4) detects the node failure, activates the corresponding processor-timer while resetting the faulty node. After a time-out, if WP1 cannot recover, it is declared faulty and controller removes it from the system. The application tasks being executed by the faulty nodes are re-assigned to other healthy nodes. However, if the faulty node recovers within a time-out, system controller puts the recovered node into the system. Inter-processor communication is the key to achieving high performance from a multiple processor system. The embedded processor system is tested and verified to handle the failure of DP memories and their interface. The results are presented for DP14 or its interface failure with WP1 and/or WP4 nodes. A fault is injected in DP14 memory module. WP1 detects the fault successfully during a message transfer as given in Figure 8. It reports the failure to IP4 by using DP24 and DP12 modules.

A temporary fault is also injected in the system controller (IP4) to verify the fault-tolerance for IP nodes. Figure 9 presents the fault detection and system recovery from the system controller failure. The backup controller (IP5) monitors IP4 and detects its failure. Then it establishes itself as a new system controller and starts diagnosing IP4. 


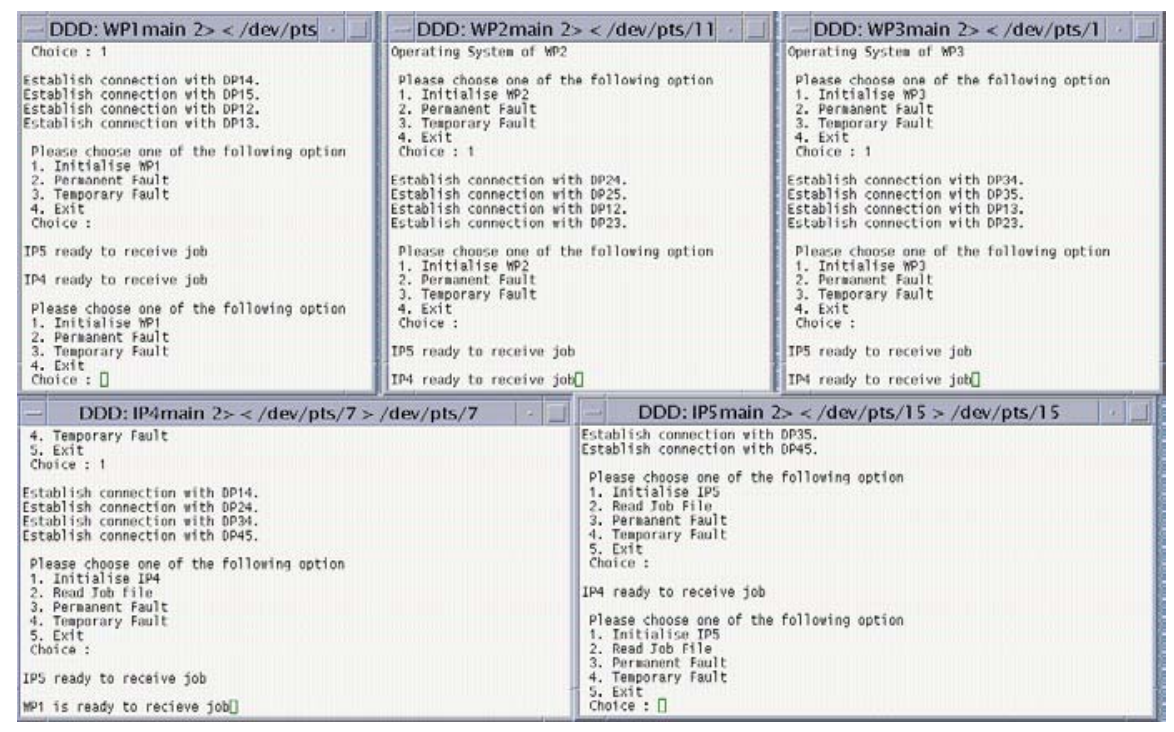

Fig. 6. Verification of system startup

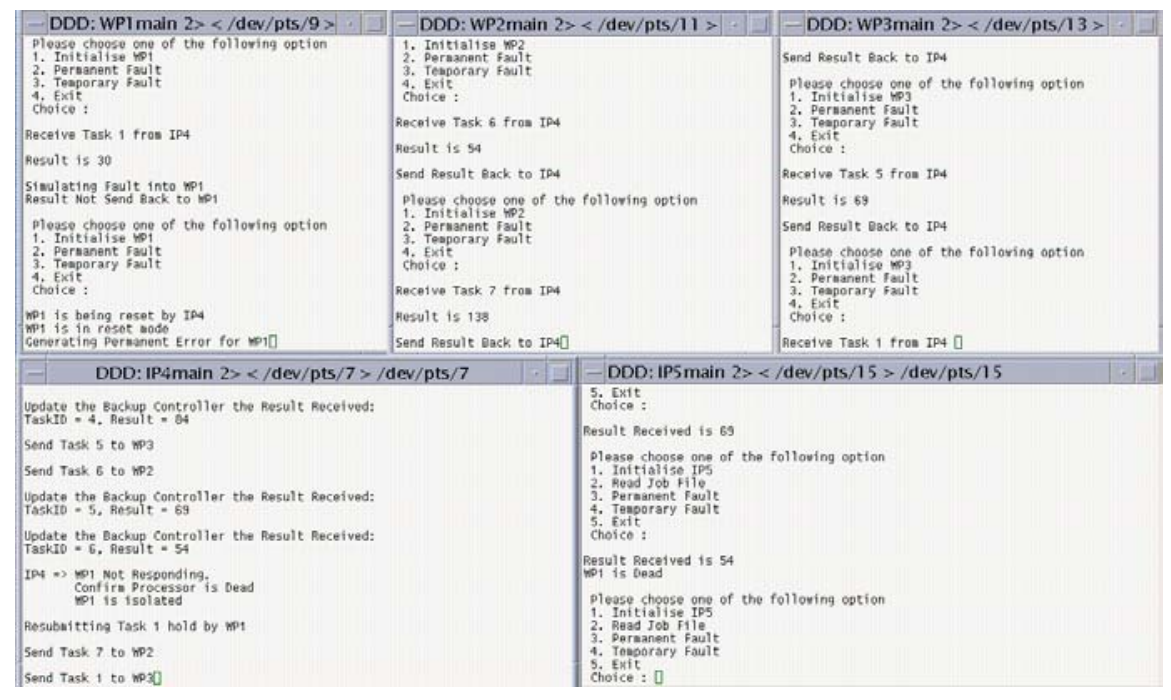

Fig. 7. System recovery from permanent fault in WP1 node

\section{CONCLUDING REMARKS}

The embedded computer architecture is modeled using Eagle $i$ tool set that provides virtual software processors to build a multiprocessor virtual hardware system. Embedded system software is executed for hardware software co-verification. The system architecture is improved to fulfill the application requirements. Hardware-software co-verification results indicate that embedded system degrades gracefully for different fault scenarios. The system would require only two fault-free communicating nodes (one in each partition) for the system to be considered operational. In the extreme case, even one healthy IP node keeps the embedded system operational at lowest level of performance. The main goal of this research is to design and develop an SOC level high performance embedded system. The system will be co-verified using Seamless from the hard real-time point of view. 


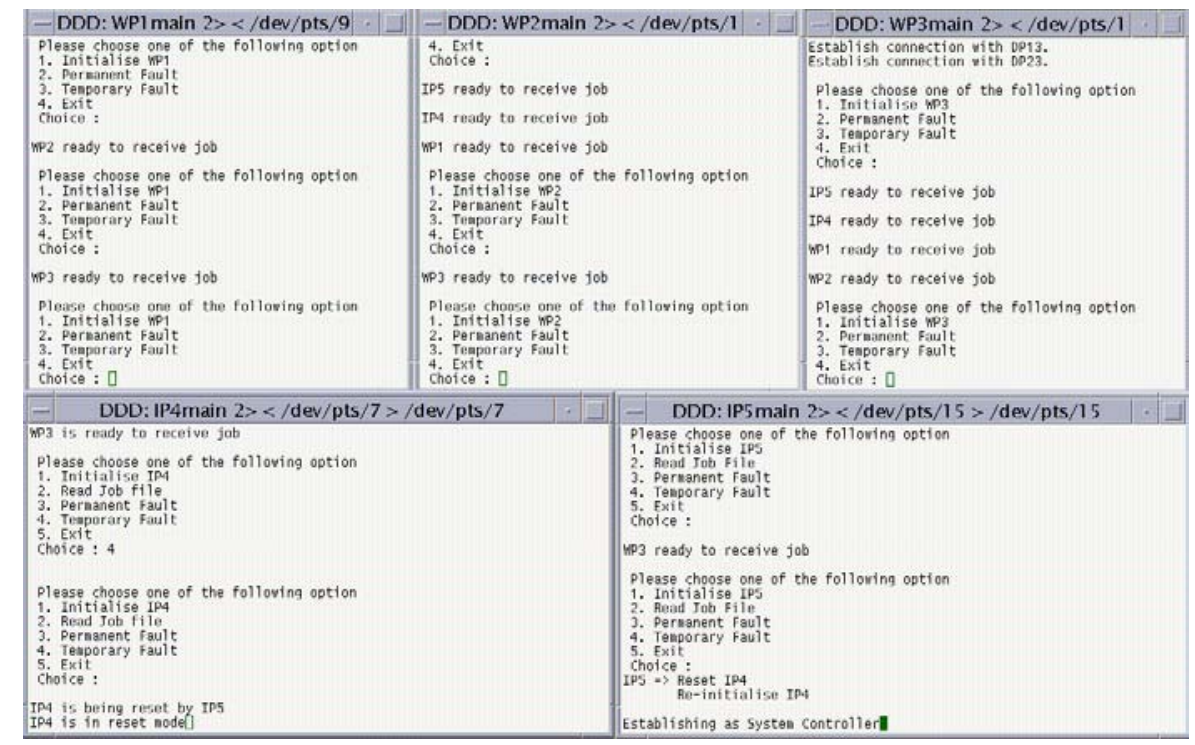

Fig. 8. Communication module DP14 failure and system recovery
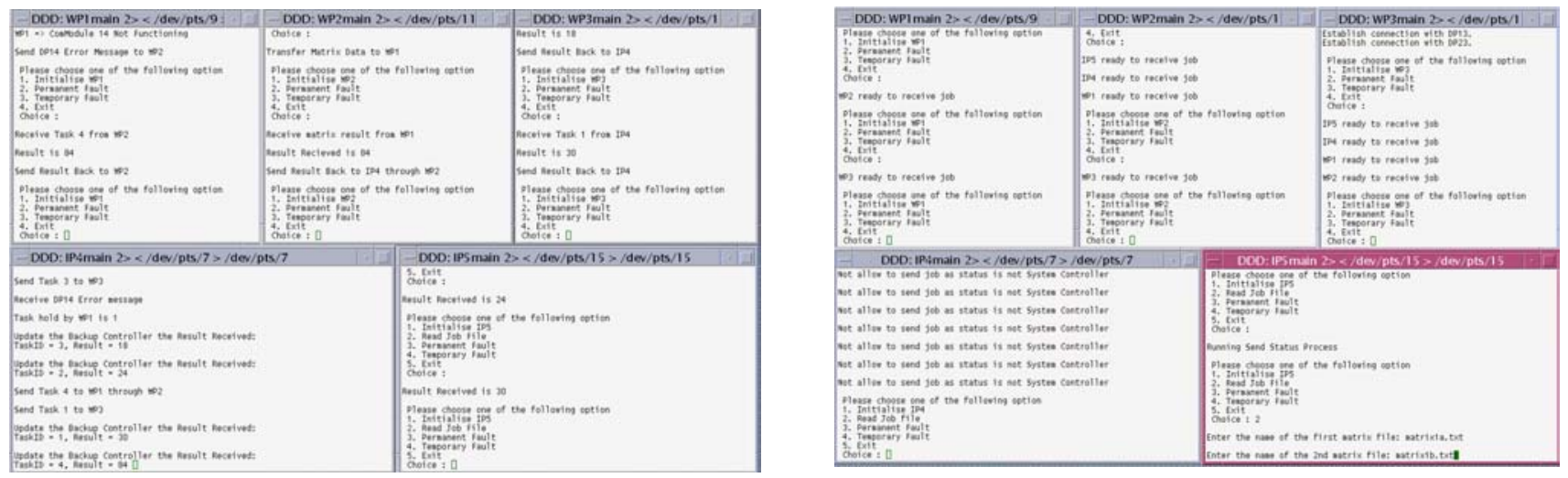

Fig. 9. Temporary fault at system controller IP4

\section{Acknowledgements}

This research is supported by a grant from NSERC Canada. The authors would also like to thank CMC for providing hardware software co-design CAD tools.

\section{References}

[1] Gul N. Khan, "Fault-tolerance evaluation of a high performance embedded computer system," SAS Research Link, vol. 4/99, Singapore, pp. 4-5, January 1999.

[2] J. C. Laprie, J. Arlat, C. Beounes and K. Kanoun, "Definition and analysis of hardware and software faulttolerant architectures," IEEE Computer, vol. 23, no. 7, pp. 39-61, 1990.

[3] M. Chiodo, P. Giusto, A. Jurecska, H. C. Hsieb, A. S. Vincentelli and L. Lavagno, "Hardware-software codesign of embedded systems," IEEE Micro, vol. 14, no. 4, pp. 2636, August 1994.
[4] F. Slomka, M. Dorfel, R. Munzenberger and R. Hofmann, "Hardware/software codesign and rapid prototyping of embedded systems," IEEE-Design and Test of Computers, vol.17, no.2, pp.28-38, April-June 2000.

[5] Gul N. Khan and Duncan F. Gillies, "Vision based navigation system for an endoscope," Image and Vision Computing, vol. 14, no. 10, pp. 763-772, 1996.

[6] G. D. Bell, R. S. Rowland, M. Rutter, M. Abu-Sada, S. Dogramadzi and C. Allen, "Colonoscopy aided by magnetic 3D imaging: Assessing the routine use of a stiffening sigmoid overtube to speed up the procedure," Medical and Biological Engineering and Computing, vol. 37, no. 5, pp.605-611, Sept. 1999.

[7] S. D'Attanasio, O. Tonet, G. Megali, M. C. Carrozza and P. Dario, "A semi-automatic handheld mechatronic endoscope with collision-avoidance capabilities," in Proc. IEEE International Conference on Robotics an Automation, pp. 1586-1591, 2000. 